\title{
CircTMCO3 Promotes Gastric Cancer Progression by Regulating miR-577/RABI4 Axis
}

\author{
Peng Yu \\ Ke Wei \\ Taimin Zhang \\ Zhenzong Tan \\ Hezhao Zhao \\ Hao Sun
}

Chongqing University Cancer Hospital Gastrointestinal Department, Chongqing, 400000, People's Republic of China
Correspondence: Hao Sun; Hezhao Zhao Chongqing University Cancer Hospital, Gastrointestinal Department, Chongqing, 400000, People's Republic of China Email sunhao68@hotmail.com; zhzalee@hotmail.com
Background: Growing evidence indicated that circRNAs played major roles in the progression of human cancer. Nevertheless, the molecular mechanism and effects of circTMCO3 in GC are still unclear.

Methods: First, qRT-PCR was used to evaluate the levels of circTMCO3 from GC tissues, GC cells, normal tissues and gastric epithelial cells. Then, the GC cells were transfected to analyze the proliferation, migration and invasion of GC cells by MTT, colony formation and transwell assays. Next, the expressions of miR-577 and RAB14 in GC tissues and cells were examined by qRT-PCR following transfection. The target interaction of circTMCO3-miR -577 and miR-577-RAB14 was explored by the dual-luciferase reporter and RNA pull-down assays. In the end, the growth and viability of GC cells were detected by MTT, colony formation and transwell assays, respectively, following the transfection of GC cells.

Results: In this research, we found circTMCO3 expressions are significantly up-regulated in GC tissues and cells compared with the normal tissues and gastric epithelial cells. We discovered that the knockdown of circTMCO3 remarkably inhibits the proliferation, migration and invasion of GC cells. Besides, through the prediction of binding sites between circTMCO3, miR-577 and RAB14, we discovered miR-577 is a target of circTMCO3 while RAB14 is a target gene of miR-577. Finally, the results demonstrate the overexpression of miR-577 and the silence of RAB14 could inhibit the effects of circTMCO3 on proliferation, migration and invasion in GC cells.

Conclusion: circTMCO3 accelerated the growth and migration of GC cells by regulating miR-577/RAB14 axis.

Keywords: gastric cancer, circTMCO3, miR-577, RAB14, KATO III, HGC-27

\section{Introduction}

Gastric cancer (GC) leads to a high mortality rate and is one of the most common malignant tumors in the world. ${ }^{1-3}$ Despite significant improvements in the diagnosis and treatment strategies for GC, the 5-year overall survival rate for patients with advanced GC is only about $30 \%{ }^{4,5}$ Surgery, adjuvant or neoadjuvant therapy are often used in conjunction with, and are the only radical treatments for GC for now. However, the molecular mechanisms that cause the occurrence, development, recurrence, and metastasis of GC are not fully understood. Therefore, finding novel potential biomarkers for early diagnosis and prediction of GC prognosis can provide a new insight for the treatment of GC.

Circular RNAs, a non-coding RNAs with a circular structure, have recently been reported in many tumors, such as bladder cancer, ${ }^{6,7}$ colorectal cancer, ${ }^{8,9}$ and cervical cancer. ${ }^{10}$ The expressions of circRNAs are abnormal in various malignant 
tumors as a diagnostic marker and therapeutic intervention target for tumors. ${ }^{11,12}$ Functionally, circRNAs can bind miRNAs and affect the binding of miRNA to target genes.

circRNAs up-regulate the target genes of miRNAs indirectly and act as a sponge for "adsorption" of miRNAs, which affects the proliferation, apoptosis, migration and invasion of cancer cells. ${ }^{13-15}$ miRNAs can pair with the mRNA bases of target genes to induce silencing complex (RISC) to degrade the mRNA or inhibit its translation. There is increasing evidence that miRNAs play critical roles in regulating tumor progressions, such as breast cancer, melanoma, and gastric cancer. ${ }^{16-18}$ circRNAs might exert their functions through targeting miRNAs.

Feiran Wang et al speculate that circTMCO3 might participate in the pathogenesis of HCC. However, the mechanisms of circTMCO3 to affect cancer progression are still unclear.

In this research, we studied the expressions of circTMCO3 in GC tissues and cell lines by using circRNAs bioinformatics and functional studies. The purpose of our study is to find out the roles and mechanisms of circTMCO3 in GC progression, and we try to conclude whether circTMCO3 could sponge miRNA to regulate the GC progression.

\section{Materials and Methods}

\section{Tissue Samples and Cell Lines}

The research involved $40 \mathrm{GC}$ patients who underwent surgery at Chongqing University Cancer Hospital. Adjacent normal tissues (ANTs), in which no obvious tumor cells were found in pathologists, were taken from $5 \mathrm{~cm}$ away from the tumor edge. GC Patients with a history of tumor therapy or other solid tumors were excluded from this research. This study was approved by the Ethics Committee for Human Research of Chongqing University Cancer Hospital and was conducted in accordance with the Declaration of Helsinki. All patients obtained informed consent.

Gastric epithelial cell line GES-1, GC cell lines MKN28, SGC-7901, KATO III, and HGC-27 were obtained from the Institute of Biochemistry and Cell Biology of the Chinese Academy of Sciences (Shanghai, China). All cell lines were cultured in 90\% RPMI-1640 medium (GIBCO) containing 10\% fetal bovine serum (FBS) and $1 \%$ penicillin-streptomycin solution
(Invitrogen, Carlsbad, CA, USA) at $37^{\circ} \mathrm{C}$ in a moist atmosphere with $5 \% \mathrm{CO} 2$ for subsequent experiments.

\section{Quantitative Real-Time Polymerase Chain Reaction (qRT-PCR)}

Total RNA was extracted using TRIzolTM reagent (Invitrogen, CA, USA). To ensure the purity of circRNAs, using RNase R (Geneseed, Guangzhou, China) to dispose the RNAs for 20 min. RNA concentration was measured by Nanodrop 2000 (Thermo, America). The qRT-PCR was performed on a 7500 Fast Real-Time PCR System (Applied Biosystems, Thermo Fisher Scientific) using the SYBR Premix Ex Taq II kit (Takara Bio, Beijing, China) to examine the relative expression of circRNAs and miRNAs. GAPDH was used to standardize circRNAs and mRNAs expression levels.

First, RNAs were reversed into cDNAs by using TaqMan Reverse-transcription. The cDNAs were synthesized using the PrimeScript RT reagent Kit (Takara, Tokyo, Japan) transcriptase, Random 6mers, RNase inhibitor, Oligo dT primer, dNTP mixture, and reaction buffer. The cycle conditions were at $95^{\circ} \mathrm{C}$ for $30 \mathrm{~s}$ (initial denaturation), followed by $95^{\circ} \mathrm{C} 5 \mathrm{~s}, 60^{\circ} \mathrm{C} 34 \mathrm{~s}, 40$ cycles. The data were analyzed by $2-\Delta \Delta \mathrm{Ct}$ method. ${ }^{19}$

Follows are the primer sequences:

circTMCO3: (Forward): 5'-GGACCCTATTATCGGA AGC-3';

(Reverse): 5'-CACCTATGGAGGCGAAGAA-3';

miR-577: (Forward): 5'-TGCGGTAGATAAAATAT TGG-3';

(Reverse): 5'-CCAGTGCAGGGTCCGAGGT-3';

GAPDH: (Forward): 5'-AGGTGAAGGTCGGAGTC AACG -3';

(Reverse): 5'-AGGGGTCATTGATGGCAACA-3';

RAB14: (Forward): 5'-TATGGCTGATTGTCCTCAC ACA-3';

(Reverse): 5'-CTGTCCTGCCGTATCCCAAAT-3';

U6: (Forward): 5'-CTCGCTTCGGCAGCACATAT ACT-3';

(Reverse): 5'-ACGCTTCACGAATTTGCGTGTC-3';

\section{Cell Transfection}

The small interfering RNAs, negative controls and miR577 inhibitors were synthesized and purchased from Genepharma Company (Shanghai, China). circTMCO3 vectors were constructed using amplified DNA fragments containing 15.16 exons of TMCO3 gene and lateral introns 
containing complementary Alu elements (GeneChem, Shanghai, China). The following vectors and oligonucleotides were transfected into KATO III and HGC-27 cells by using Lipofectamine 3000 (Invitrogen, CA, USA) for $24 \mathrm{~h}$. si-circTMCO3: 5'-GGGAGCTAAACCCGTTTAA-3'; si-RAB14: 5'-CCGCTGCGTCCGCACTCCT-3'; si-NC: 5'-UUCUCCGAACGUGUCACGU-3'; miR-NC: 5'-GUGGAUUUUCCUCUAUGAUUU-3';

\section{Cell Proliferation Assay - MTT Assay}

Trypsin was used to digest the logarithmic cells which were collected for centrifugation to prepare cell suspension. The cell suspension was mixed and added $100 \mu \mathrm{L}$ cells into each well of 96-well-plate. The cells were cultured at $37^{\circ} \mathrm{C}$ in an incubator of $5 \% \mathrm{CO} 2$ until the whole bottom was monolayer covered by them. Then, $10 \mu \mathrm{L}$ MTT solution ( $5 \mathrm{mg} / \mathrm{mL}, 0.5 \%$ MTT) was added to each well, and the cells continued to be cultured for $4 \mathrm{~h}$. After washing with PBS 2-3 times, the culture solution containing MTT was added. $150 \mu \mathrm{L}$ dimethyl sulfoxide was added to each well and shook them at a low speed for $10 \mathrm{~min}$ to completely dissolve the crystals. The absorbance values of each well were measured at OD490 nm.

\section{Colony Formation Assay}

Trypsin was used to digest logarithmic growth cells in each experimental group, and the cell suspension was prepared in a complete medium. Then, we counted the cells, removed the medium, and washed the cells with PBS. We added trypsin to terminate the digestion and obtain the cell precipitation after centrifugation. The cells were implanted in 6-well plates, each group 500-1000 cells/well. The cells were shaken gently and put in an incubator for the next culture. We replaced the cell culture medium every $3 \mathrm{~d}$ and observed the cell state. The microscope was used to monitor the size of colony cells. When the individual colonies were grown to a suitable size, we added $1000 \mu \mathrm{L}$ pure, impurity-free crystal violet dye into each well to dye for $2 \mathrm{~min}$.

\section{In vitro Cell Migration and Invasion Assay -Transwell Assay}

The transwell assay was used to detect the invasion and migration ability of the GC cells. After $48 \mathrm{~h}$ transfection, the cell culture transwell inserts ( 8 -mm pore size; Falcon; BD Biosciences) were put into the 48 -well plates to generate the departed upper and lower chambers. Then, we cultured the upper $1 \mathrm{~h}$ at $37^{\circ} \mathrm{C}$ for gelation. We hydrated the membrane with FBS $2 \mathrm{~h}$ prior. RPMI-1640 $(600 \mu \mathrm{L})$ with $10 \%$ FBS and $1 \times 105$ cells/well was put into the lower and upper chamber separately. After 24 hours of culture, we counted the number of invaded cells under a microscope to detect the migration ability. Furthermore, after repeating the above steps, we used Matrigel (BD Biosciences, San Jose, CA) to pre-coat the upper part of the membrane to detect the invasion ability.

\section{RNA Probe Pull-Down Assay}

To demonstrate the interaction between circTMCO3, miR577, and RAB14, this experiment performed RNA pulldown assay. Biotinylated probe was designed to bind to the junction region of circTMCO3 or miR-577. We also use oligonucleotide probe as a negative control. About $1 \times 107$ cells were lysed in a lysis buffer and incubated with $3 \mu \mathrm{g}$ biotinylated probe for $2 \mathrm{~h}$. Cell lysates were incubated with streptavidin magnetic beads (Life Technologies, Gaithersburg, MD, USA) for $4 \mathrm{~h}$ to pull down the biotin-conjugated RNA complex. The lysis buffer was used to wash the complex 5 times. Then, the binding miRNA in the pull-down complex was extracted with TRIzol reagent and analyzed by qRT-PCR.

\section{Dual-Luciferase Reporter Assay}

Targets analysis of circTMCO3 and miR-577 was conducted on the biological prediction website microRNA. org, respectively.

circTMCO3 sequences containing miR-577 binding sites and RAB14 3' untranslated region (UTR) were cloned into luciferase reporter vectors (Promega, Madison, WI, USA) to form wild-type (wt) luciferase reporter vectors wtcircTMCO3 and RAB14 3' UTR-wt. The mutant (mut) luciferase reporter vector constructs, mut-circTMCO3 and RAB14 3'UTR-mut, were created by mutating the binding sites pf miR-577. For dual-luciferase reporter assay, KATO III and HGC-27 cells were co-transfected with these constructs and miR-577 mimic or mimic control. After $48 \mathrm{~h}$, luciferase activity was measured by using a dual-luciferase reporter assay system (Promega).

\section{Statistical Analysis}

SPSS v.19.0 (IBM, SPSS, Chicago, IL, USA) was used for statistical analysis. Comparison between the two groups was performed by Student's $t$-test and one-way ANOVO. And a p-value less than 0.05 was considered statistically significant. 


\section{Result}

\section{circTMCO3 is Up-Regulated in GC}

\section{Tissues and Cells}

To study the role of circTMCO3 in the pathogenesis of GC, we examined the expression levels of circTMCO3 in the tumor tissues, GC cells, adjacent normal tissues, and GES-1 cells. The qRT-PCR results showed that the expression levels of circTMCO3 in GC tissues were significantly higher than that in normal tissues (Figure $1 \mathrm{~A}, \mathrm{P}<0.01$ ). The qRT-PCR analysis also indicated that the expressions of circTMCO3 were remarkably increased in KATO III and HGC-27 cell lines compared to GES-1 cells (Figure 1B, $\mathrm{P}<0.01$ ).

\section{Knockdown of circTMCO3 Inhibits Cell Proliferation, Migration and Invasion}

Next, to further explore the role of circTMCO3 in GC, we inhibited circTMCO3 in KATO III and HGC-27 cells. Thus, qRT-PCR was used to demonstrate the effect. The results showed that the silence of circTMCO3 could effectively reduce the expressions of circTMCO3 (Figure 2A, $\mathrm{P}<0.01$ ). Next, MTT and colony formation assays were performed to determine GC cells' growth after transfecting with circTMCO3. As shown in Figure 2B, the cell viability of sicircTMCO3 group was significantly decreased after transfecting $72 \mathrm{~h}$. $(\mathrm{P}<0.01)$. The results showed that the knockdown of circTMCO3 significantly inhibited the cell proliferation of KATO III and HGC-27 cells (Figure 2C, P<0.01). Additionally, transwell assays were used to investigate the migration and invasion of GC cells. We found that knockdown of circTMCO3 could inhibit the migration and invasion of
KATO III and HGC-27 cells in vitro (Figure 2D and E, $\mathrm{P}<0.01)$.

\section{circTMCO3 Acts as a Sponge of miR-577 in GC Cells}

First, we performed qRT-PCR to detect the expressions of miR577 in tumor tissues and adjacent normal tissues. The results indicated that the expression levels of miR-577 in GC tissues were significantly lower than that in normal tissues (Figure 3E, $\mathrm{P}<0.01$ ). Figure $3 \mathrm{~A}$ demonstrates the complementary base sequences between circTMCO3 and miR-577. Second, to determine the interaction between circTMCO3 and miR-577, we performed luciferase activity reporter and RNA pull-down assays. The consequence showed that the overexpression of miR-577 in wt-circTMCO3 declined the luciferase activity of KATO III and HGC-27 cells more than 50\%, while mutcircTMCO3 almost eliminated this effect (Figure 3B, $\mathrm{P}<0.01$ ). Subsequently, we designed the circTMCO3 overexpressing and siRNA vector and transfected them into KATO III and HGC-27 cells. circTMCO3 overexpression lowered the level of miR577, while the circTMCO3 knockdown augmented the same (Figure $3 \mathrm{C}, \mathrm{P}<0.01$ ). Then, circTMCO3 probe and biotin miR577-probe were used to perform RNA pull-down assay. The results showed circTMCO3 probe could enrich miR-577 in the circTMCO3-RNA complex. Simultaneously, the biotin-miR -577 probe has shown the same effects (Figure 3D, $\mathrm{P}<0.05$ ).

\section{miR-577 Reversed the Function of circTMCO3 in GC Cells}

Being a target of circTMCO3, we predicted that miR-577 had been involved in the biological mechanism of GC

B

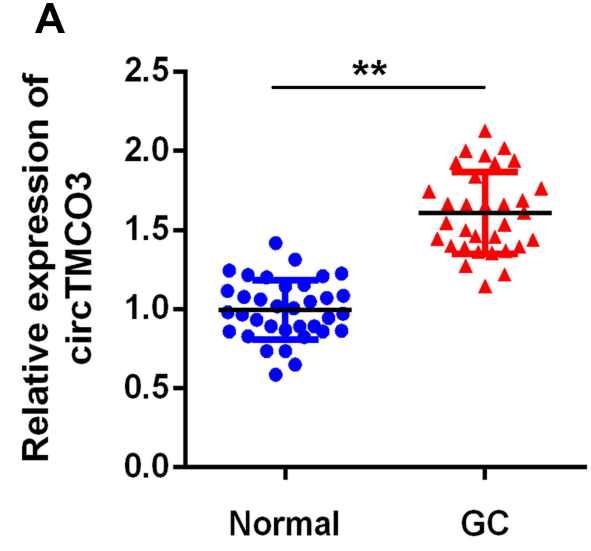

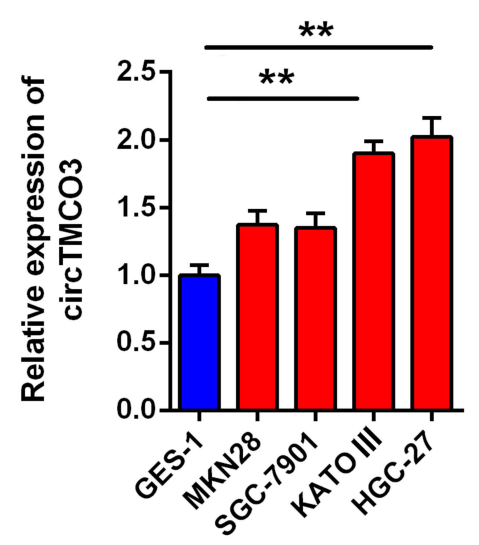

Figure I circTMCO3 is down-regulated in gastric cancer $(\mathbf{A})$. The expressions of circTMCO3 were detected in GC tissues and normal tissues by qRT-PCR (**P $<0.0 \mathrm{I})(\mathbf{B})$. The expressions of circTMCO3 were detected in normal gastric mucosal cell line GES-I and GC cell lines (MKN28, SGC-790I, KATO III and HGC-27). circTMCO3 expressions in KATO III and HGC-27 cells were significantly higher than that in GES-I cell $(* *$ P $<0.0 \mathrm{I})$. 
A

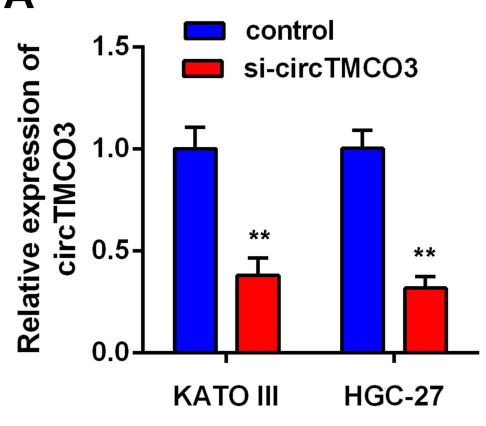

C

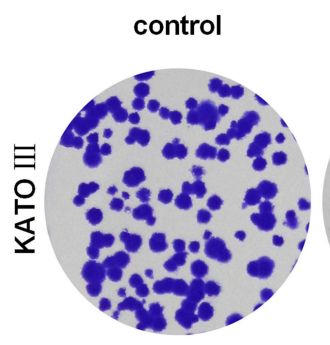

D control

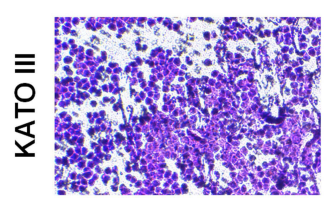

E

control

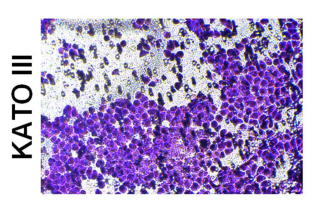

B

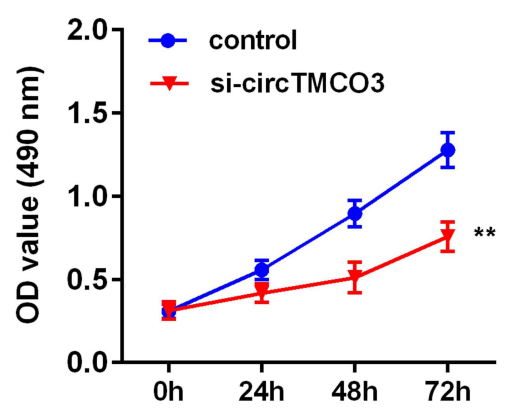

HGC-27

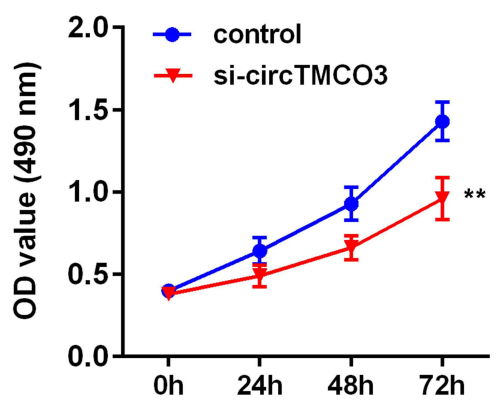

$\square$ control

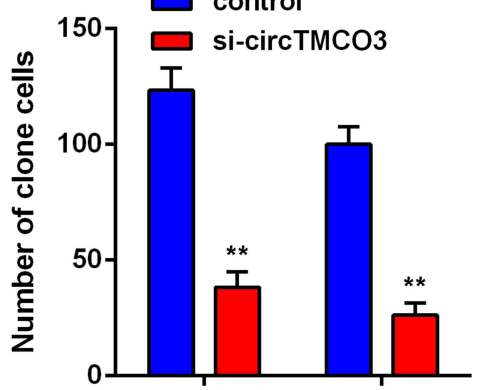

KATO III HGC-27

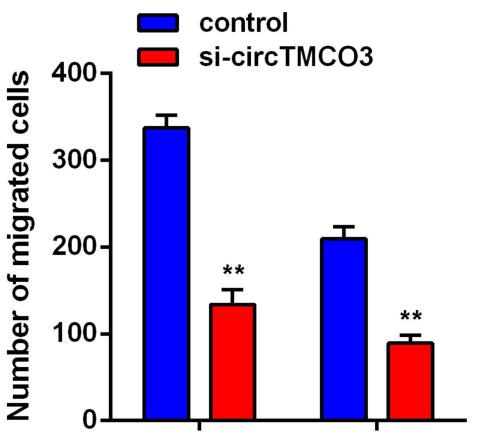

KATO III HGC-27

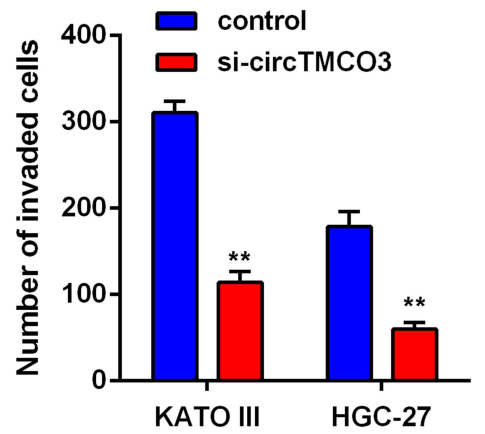

Figure 2 circTMCO3 inhibits the proliferation, migration and invasion in GC cells (A). qRT-PCR was used to evaluate the expression of circTMCO3 in KATO III and HGC27 cells $(* * \mathrm{P}<0.0 \mathrm{I})(\mathbf{B})$. The viability of KATO III and HGC-27 cells transfected with si-circTMCO3 were detected at 0,12,24,28 and 72h by MTT assay (**P<0.0 I) (C). The proliferation transfected with si-circTMCO3 or vector were detected by colony formation assay $(* * P<0.01)(\mathbf{D}$ and E). The migration and invasion of KATO III and HGC-27 cells transfected with si-circTMCO3 or vector were analyzed by transwell assay $(* * \mathrm{P}<0.01)$.

cells as well. miR-577 expression was significantly increased in KATO III and HGC-27 cells after transfected with si-circTMCO3 (Figure 3E $\mathrm{P}<0.01$ ). Co-transfection with miR-577 inhibitor and circTMCO3 suppressing vector apparently decreased the level of miR-577 in comparison with circTMCO3 suppressing group (Figure 4A $\mathrm{P}<0.05)$. By means of the function studies, we, at first, discovered that miR-577 inhibitor reversed the effect of 
A

circTMCO3 wt: 5' GgGAAUAUCUGCCUUUAUCUU 3'

miR-577: 3' guccaugguUAUAAAAUAgAU 5'

circTMCO3 mut: 5' GgGAAUAUCUGCCUUAUAGAU 3'

C

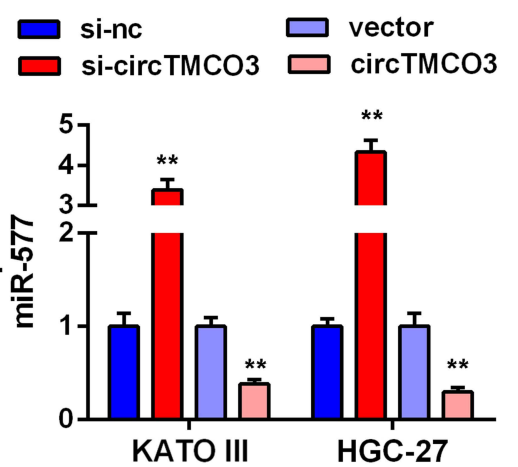

D

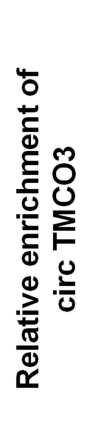

IIIII|

B

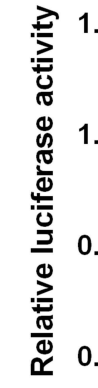

E

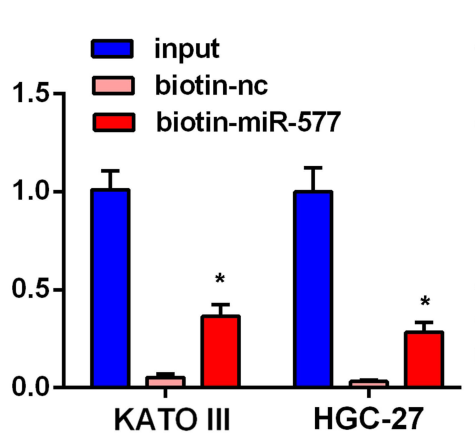

mimic NC

miR-577 mimic

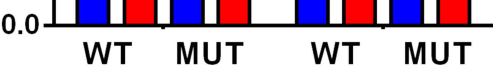

KATO III HGC-27

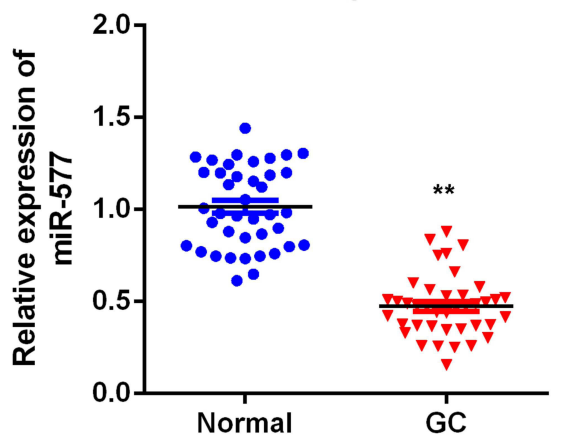

Figure 3 circTMCO3 acts as a sponge of miR-577 in GC cells (A). Bioinformatics predicted miR-577 has the binding sites of circTMCO3. (B) Dual-luciferase reporter assay was indicated that transfection with miR-577 mimic significantly reduced the luciferase activity of the reporter containing wt- cricTMCO3 in KATO III and HGC-27 cells $(* * \mathrm{P}<0.0 \mathrm{I})$. (C) After the knockdown and overexpression of circTMCO3, miR-577 expression levels were detected (**P<0.0I). (D) RNA pull-down assay: miR-577 was enriched in the circTMCO3-RNA complex and miR-577 was pulled down to detect the content of circTMCO3 ( $* \mathrm{P}<0.05)$. (E) The expressions of miR-577 in GC tissues and normal tissues were detected by $\mathrm{q}-\mathrm{RT}-\mathrm{PCR}(* * \mathrm{P}<0.0 \mathrm{l})$.

circTMCO3 not only on the cell proliferation (Figure 4B and C) but also on migration (Figure 4D) as well as invasion (Figure 4E) of KATO III and HGC-27 cells.

\section{RABI4 is a Target Gene of miR-577}

Additionally, we tried to detect the target gene of miR-577 through Microarray analysis. The results showed miR-577 targeted RAB14 (Figure 5A). First, we performed qRT-PCR to detect the expressions of RAB14 in tumor tissues and adjacent normal tissues. The results indicated that the expression levels of RAB14 in GC tissues were significantly higher than that in normal tissues (Figure 5E, $\mathrm{P}<0.01$ ). Secondly, to determine the interaction between RAB14 and miR-577, we performed luciferase activity reporter and RNA pull-down assays. The consequence showed that the overexpression of miR-577 in wt-RAB14 declined the luciferase activity of KATO III and HGC-27 cells by more than $50 \%$, while mutRAB14 almost eliminated this effect (Figure 5B, $\mathrm{P}<0.01$ ). In addition, qRT-PCR analysis further confirmed that miR-577 overexpression decreased the level of RAB14, while knockdown of miR-577 increased that of RAB14 (Figure 5C, $\mathrm{P}<0.01)$. Then, RNA-pull down experiments were carried out; the results indicated that miR-577 directly target RAB14 (Figure 5D, $\mathrm{P}<0.05$ ).

\section{The Silence of RABI4 Inhibits the Oncogenic Effects of circTMCO3}

Finally, to further detect whether the effects of circTMCO3 in GC were depended on miR-577 and RAB14, we added miR-577 mimic and RAB14 into KATO III and HGC-27 cells. Co-transfection with miR-577 and RAB14 vector apparently decreased the level of RAB14 in comparison with miR-577 mimic group (Figure $6 \mathrm{~A}, \mathrm{P}<0.05$ ). MTT and colony formation assays were performed to determine the growth of GC cells after transfection. As is shown in Figure 6B, the cell viability of miR-577 mimic group is significantly decreased after transfecting $72 \mathrm{~h}$. The results showed that RAB14 reversed the effect of miR-577 not only the cell proliferation (Figure 6C) but also migration (Figure 6D), as well as invasion (Figure 6E) of KATO III and HGC-27 cells. The consequence showed that both the overexpression of miR-577 and RAB14 could reduce the inhibitory effect of circTMCO3 on the growth and vitality of KATO III and HGC-27 cells.

\section{Discussion}

In this research, we found circTMCO3 is up-regulated in GC. The study revealed that circTMCO3 played an 

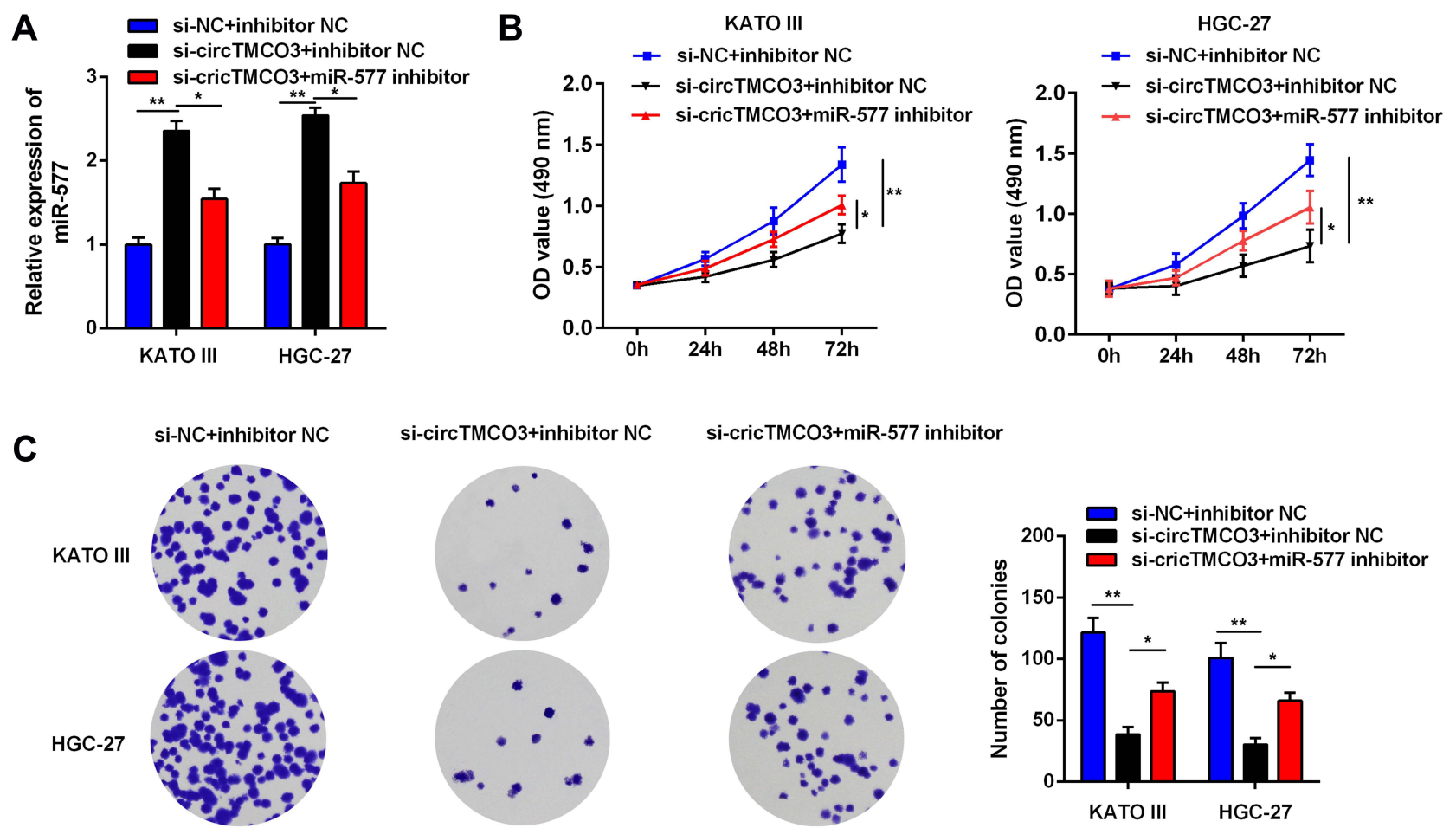

si-circTMCO3+inhibitor NC

si-cricTMCO3+miR-577 inhibitor

D
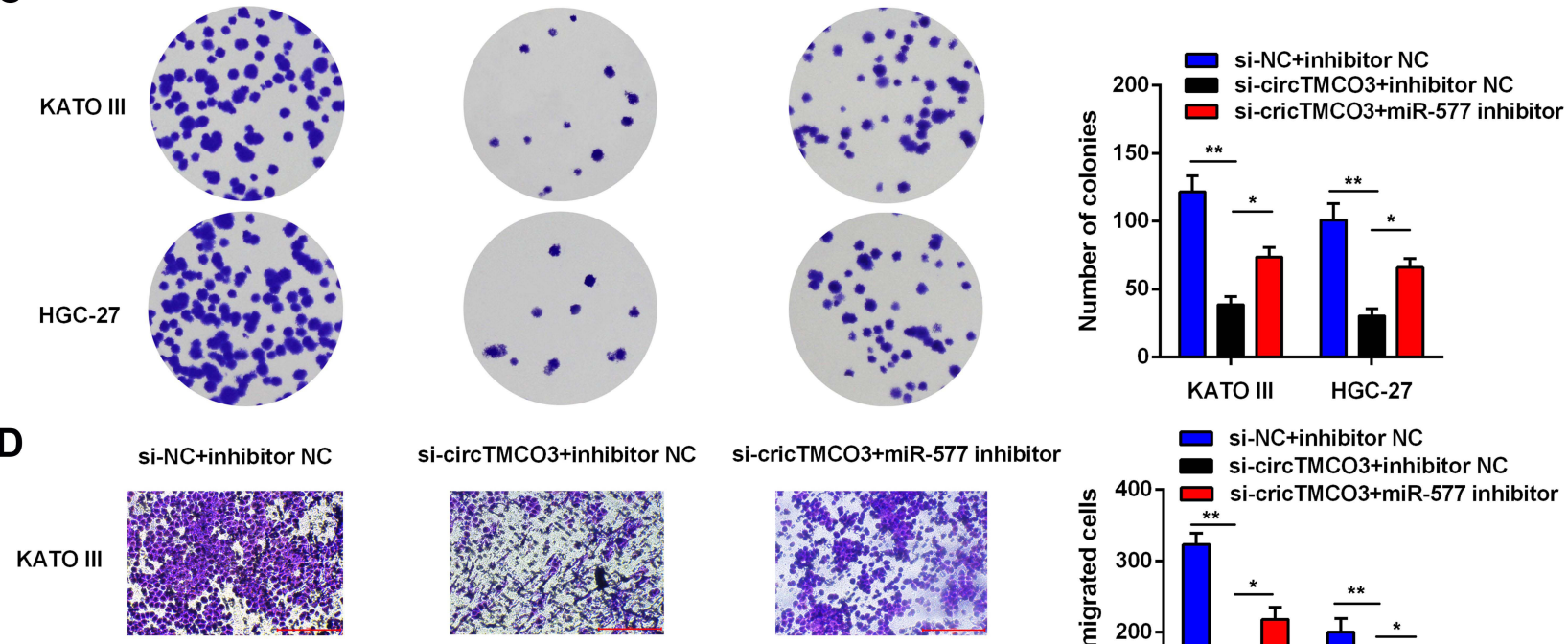

si-circTMCO3+inhibitor NC
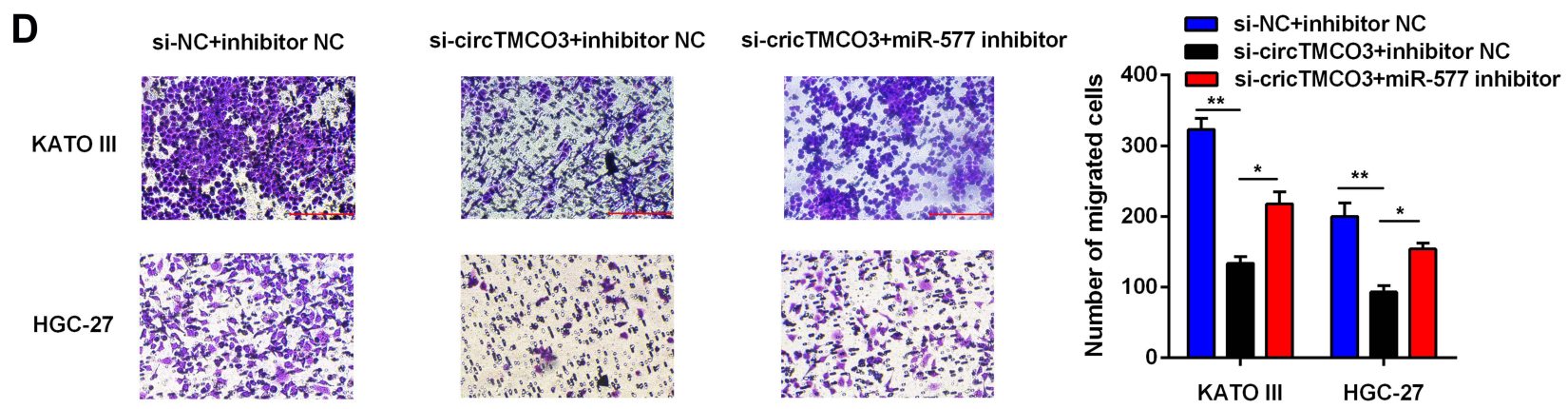

E
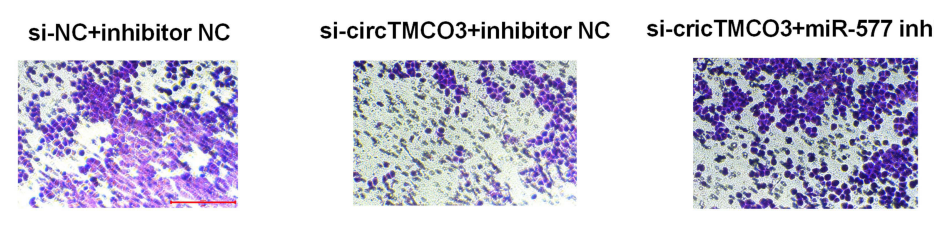

HGC-27
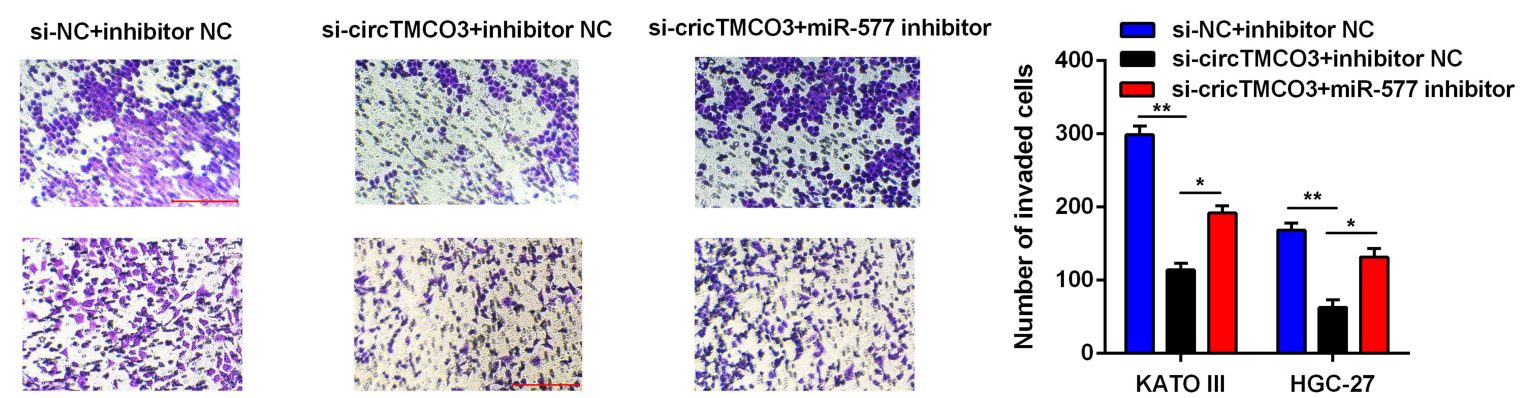

Figure 4 miR-577 reversed the function of circTMCO3 in GC cells. (A) q-RT-PCR was used to evaluate the expression level of miR-577 to confirm the influence of circTMCO3 supression on the level of miR-577 $(* \mathrm{P}<0.05$, $* * \mathrm{P}<0.0 \mathrm{I})$. (B) The viability of transfected KATO III and HGC-27 cells were detected at $0,12,24,28$ and $72 \mathrm{~h}$ by MTT assay $(* \mathrm{P}<0.05, * * \mathrm{P}<0.0 \mathrm{I}) .(\mathbf{C})$. Colony formation assay was performed to evaluate the colony formation ability of KATO III and HGC-27 cells $(* \mathrm{P}<0.05$, $* * \mathrm{P}<0.0 \mathrm{I})$. (D and E). Transwell assay was used to investigate the migration and invasion of KATO III and HGC-27 cells ( $* \mathrm{P}<0.05, * * \mathrm{P}<0.0 \mathrm{I})$.

essential role by regulating miR-577/RAB14 axis to promote the proliferation, migration, and invasion of $\mathrm{GC}$ cells.

On the other hand, DAI et al suggested that in the GEO database, the circRNAs chip found the molecular characteristics and mechanisms of circRNAs in most GC patients have not yet been elucidated. ${ }^{19}$ Moreover, circRNAs expressed stably and took part in the progression of GC. ${ }^{20}$ Several circRNAs have been found to be associated with GC tumorigenesis or metastasis, such as hsa-circ -0008035 , hsa-circ-0000670, and hsa-circ-0003159. ${ }^{21-23}$ In the present study, we used the GEO database 
A

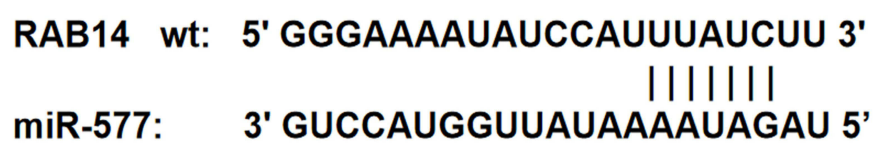

RAB14 mut: 5' GGGAAAAUAUCCAUUAUAGAU 3'
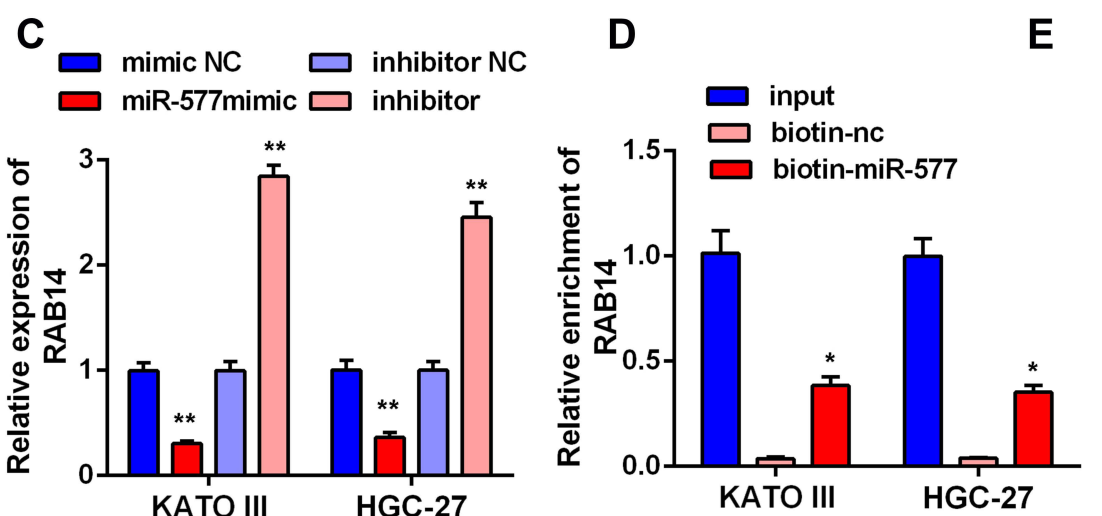

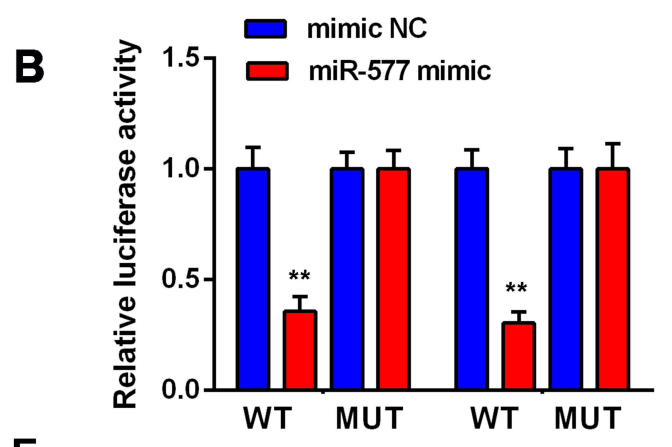

KATO III

HGC-27

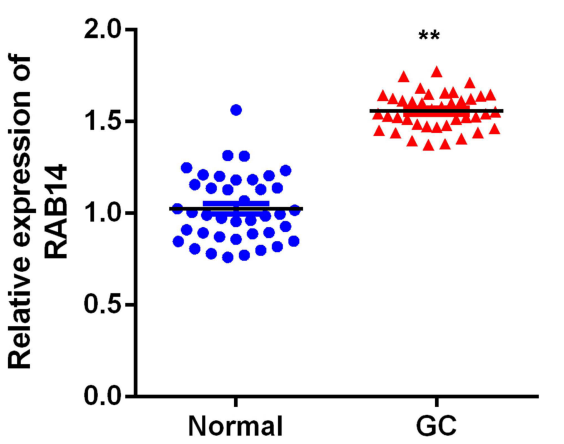

Figure 5 RABI4 is regulated by miR-577 in GC cells. (A) The complementary base sequences between RABI4 and miR-577. (B) Dual-luciferase reporter assay indicated that transfection with miR-577 mimic significantly reduced the luciferase activity of the reporter containing wt-RABI4 in KATO III and HGC-27 cells (**P<0.0I). (C) The levels of RABI4 were measured in KATO III and HGC-27 cells after transfection (**P<0.0I). (D) RNA pull-down assay: miR-577 was pulled down to detect the content of RABI4 (*P<0.05). (E) The expressions of RABI4 in tumor tissues and adjacent normal tissues $(* * \mathrm{P}<0.0 \mathrm{I})$.

circRNAs chip to screen circTMCO3, which significantly differs in GC. We verified that circTMCO3 is significantly up-regulated in GC tissues and cells compared to normal tissues and gastric epithelial cells. Moreover, after transfecting the GC cells with circTMCO3, the results of MTT, colony formation, and transwell assays showed that knockdown of circTMCO3 remarkably inhibits cell proliferation, invasion, and metastasis. In conclusion, the expressions of circTMCO3 are negatively correlated with the progression of $\mathrm{GC}$.

Besides, according to the previous papers, we found circTMCO3 can target miR-577 to regulating tumor progressions. $^{24}$ This experiment also explored whether circTMCO3 regulates GC progression by targeting miR577. circTMCO3 directly targeted miR-577 in GC cells using RNA pull-down and luciferase activity reporter assays. Consequently, we discovered that the overexpression of miR-577 could reverse the oncogenic effects of circTMCO3.

As we were known, miRNAs can regulate the behaviors of cells by combining them with the target genes. This experiment also analyzed the target gene of miR-577 and found that miR-577 targeted RAB14 in GC cells. At the same time, Bo Guo et al suggest that RAB14 presents a significantly up- regulated expression among the paired tissue samples and cell lines in gastric cancer. ${ }^{25}$ However, the expressions and functions of RAB14 gene in human GC have not been reported. Therefore, to further study whether RAB14 or miR-577 is necessary for the functions of circTMCO3, we overexpressed miR-577 or RAB14 in circTMCO3-overexpressed KATO III and HGC-27 cells. Colony formation and transwell assays were used to demonstrate that either the overexpression of miR-577 or RAB14 can reverse the oncogenic effects of circTMCO3. The present study indicated that overexpression of cricPTK2a and inhibition of miR-577 could both promote the expressions of RAB14.

\section{Conclusion}

In other words, our results proved that circTMCO3 may promote proliferation, migration, and invasion of GC cells by regulating the miR-577/RAB14 axis. The results might indicate a new target for GC therapy. These findings may provide new methods and targets for the treatments of GC.

\section{Ackwoledgments}

This research did not receive any specific grant from funding agencies in the public, commercial, or not-forprofit sectors. 


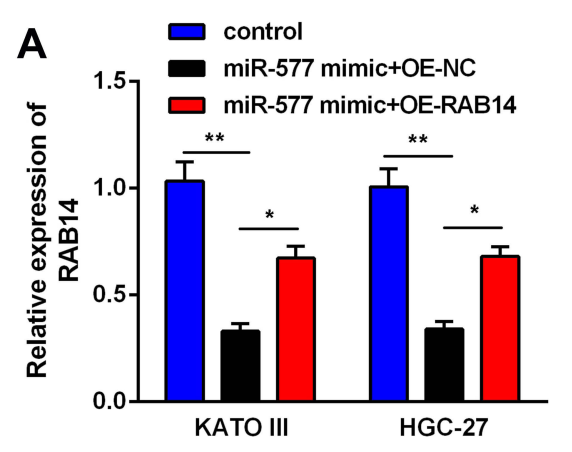

C

KATO III

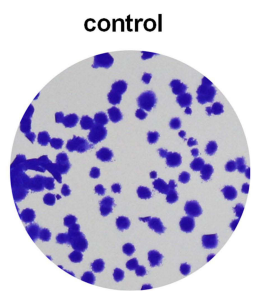

HGC-27

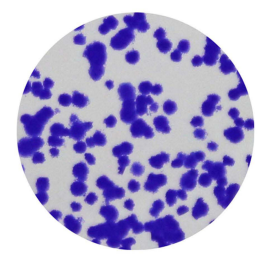

D

KATO III

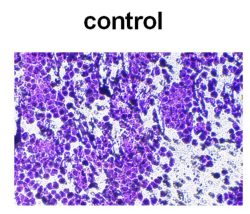

HGC-27

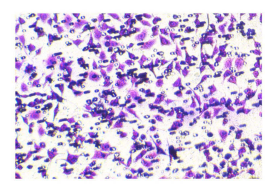

E

KATO III

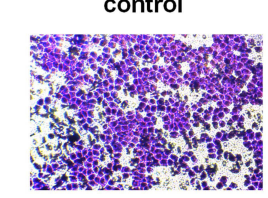

HGC-27

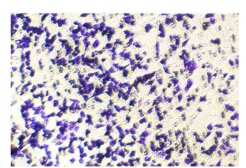

B

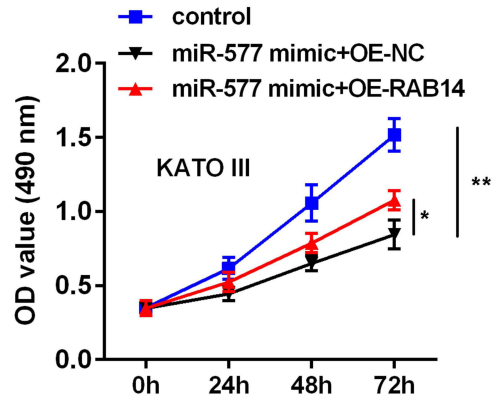

miR-577 mimic+OE-NC
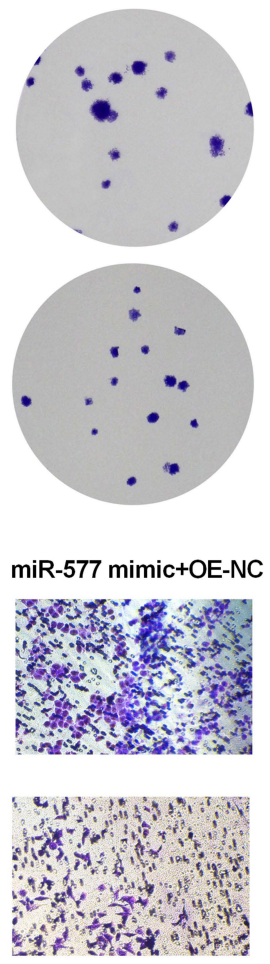

miR-577 mimic+OE-NC
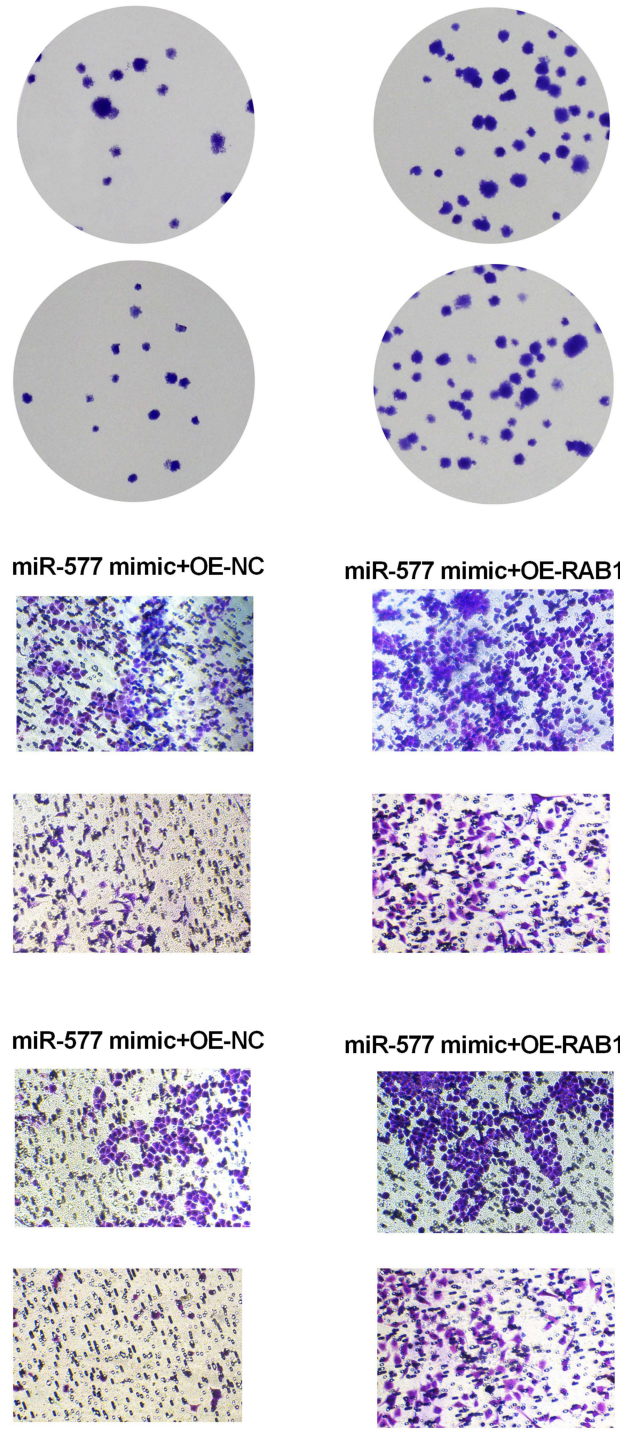

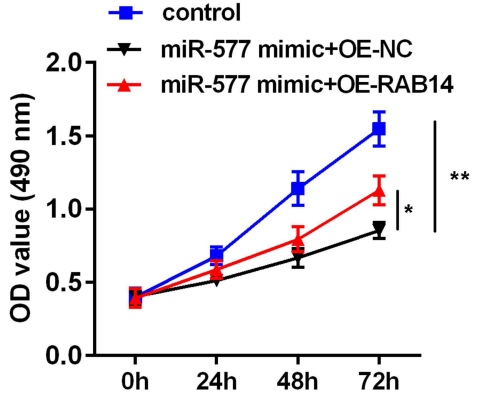

miR-577 mimic+OE-RAB14
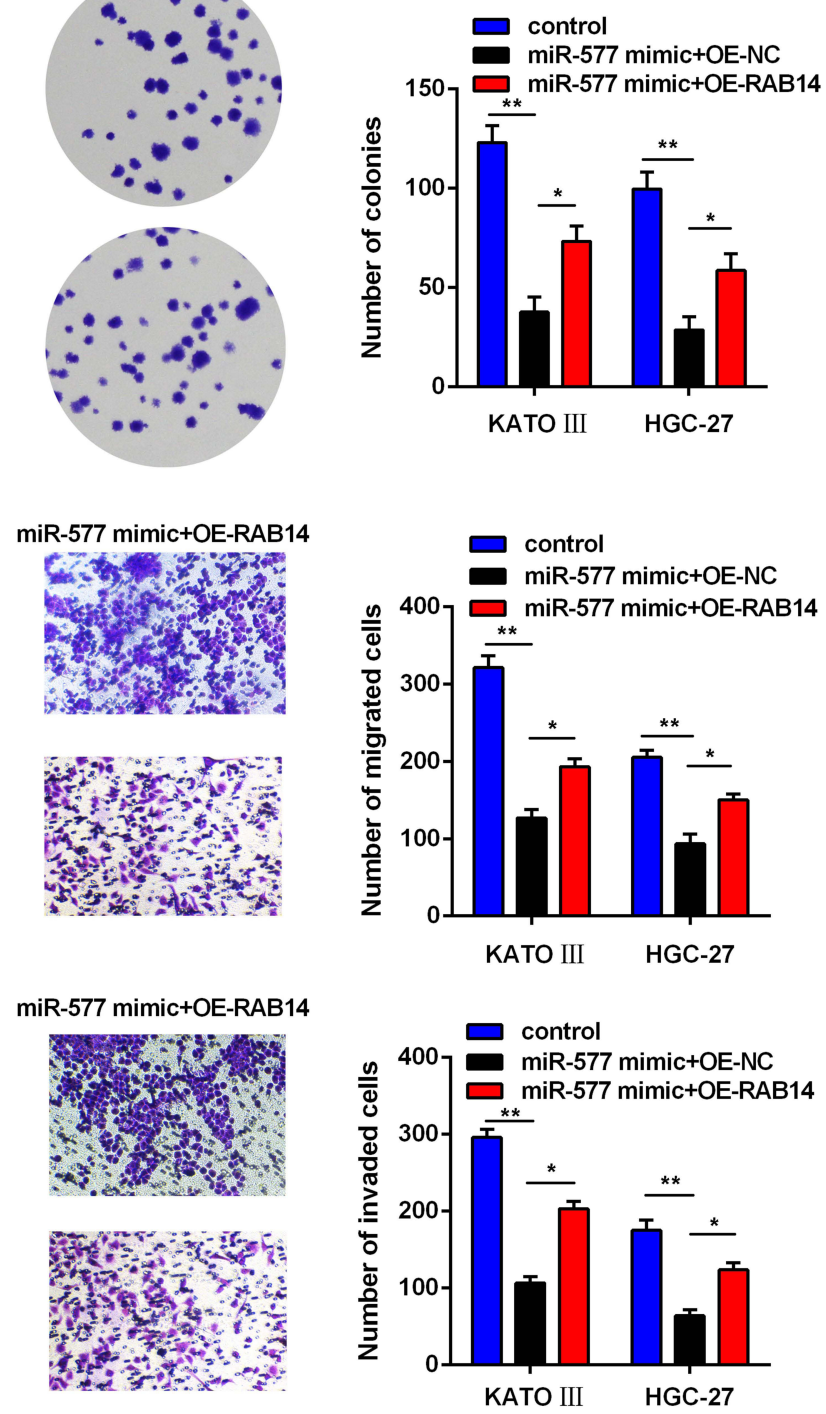

Figure 6 Silence of RABI4 reverses the anti-tumor effects of circTMCO3. (A) q-RT-PCR was used to evaluate the expression level of RABI4 to confirm the influence of miR-577 mimic $(* P<0.05, * * P<0.01)$. (B and $\mathbf{C})$. MTT and colony formation assays were performed to determine the growth of GC cells after transfection $(* P<0.05$, **P<0.0I). ( $\mathbf{D}$ and $\mathbf{E})$. The migration and invasion ability were detected in KATO III and HGC-27 cells after transfection ( $* \mathrm{P}<0.05$, **P<0.0I).

\section{Author Contributions}

All authors made a significant contribution to the work reported, whether that is in the conception, study design, execution, acquisition of data, analysis and interpretation, or in all these areas; took part in drafting, revising or critically reviewing the article; gave final approval for 
the version to be published; have agreed on the journal to which the article has been submitted; and agreed to be accountable for all aspects of the work.

\section{Disclosure}

The authors declare no competing of interests.

\section{References}

1. Jemal A, Bray F, Center MM, Ferlay J, Ward E, Forman D. Global cancer statistics. CA Cancer J Clin. 2011;61(2):69-90. doi:10.3322/ caac. 20107

2. Smyth EC, Nilsson M, Grabsch HI, van Grieken NC, Lordick F. Gastric cancer. Lancet. 2020;396(10251):635-648.

3. Karimi P, Islami F, Anandasabapathy S, Freedman ND, Kamangar F. Gastric cancer: descriptive epidemiology, risk factors, screening, and prevention. Cancer Epidemiol Biomarkers Prev. 2014;23 (5):700-713. doi:10.1158/1055-9965.EPI-13-1057

4. Ajani JA, Lee J, Sano T, Janjigian YY, Fan D, Song S. Gastric adenocarcinoma. Nat Rev Dis Primers. 2017;3:17036. doi:10.1038/ nrdp. 2017.36

5. Rugge M, Genta RM, Di Mario F, et al. Gastric cancer as preventable disease. Clin Gastroenterol Hepatol. 2017;15(12):1833-1843. doi:10.1016/j.cgh.2017.05.023

6. Han J, Wang JZ, Yang X, et al. METTL3 promote tumor proliferation of bladder cancer by accelerating pri-miR221/222 maturation in m6A-dependent manner. Mol Cancer. 2019;18(1):110. doi:10.1186/ s12943-019-1036-9

7. Li B, Xie F, Zheng FX, Jiang GS, Zeng FQ, Xiao XY. Overexpression of CircRNA BCRC4 regulates cell apoptosis and MicroRNA-101/EZH2 signaling in bladder cancer. J Huazhong Univ Sci Technolog Med Sci. 2017;37(6):886-890. doi:10.1007/ s11596-017-1822-9

8. Li XN, Wang ZJ, Ye CX, Zhao BC, Li ZL, Yang Y. RNA sequencing reveals the expression profiles of circRNA and indicates that circDDX17 acts as a tumor suppressor in colorectal cancer. $J$ Exp Clin Cancer Res. 2018;37(1):325. doi:10.1186/s13046-018-1006-x

9. Yang H, Li X, Meng Q, et al. CircPTK2 (hsa_circ_0005273) as a novel therapeutic target for metastatic colorectal cancer. Mol Cancer. 2020;19(1):13. doi:10.1186/s12943-020-1139-3

10. Ou R, Mo L, Tang H, et al. circRNA-AKT1 Sequesters miR-942-5p to Upregulate AKT1 and promote cervical cancer progression. Mol Ther Nucleic Acids. 2020;20:308-322. doi:10.1016/j. omtn.2020.01.003

11. Yang F, Liu DY, Guo JT, et al. Circular RNA circ-LDLRAD3 as a biomarker in diagnosis of pancreatic cancer. World J Gastroenterol. 2017;23(47):8345-8354. doi:10.3748/wjg.v23.i47.8345
12. Fernandez G, Biondi J, Castro S, Agamenonni O. Pupil size behavior during online processing of sentences. J Integr Neurosci. 2016;15 (4):485-496. doi:10.1142/S0219635216500266

13. Chen Q, Liu T, Bao Y, et al. CircRNA cRAPGEF5 inhibits the growth and metastasis of renal cell carcinoma via the miR-27a-3p/ TXNIP pathway. Cancer Lett. 2020;469:68-77. doi:10.1016/j. canlet.2019.10.017

14. Zhong W, Yang W, Qin Y, et al. 6-Gingerol stabilized the p-VEGFR2/VE-cadherin/beta-catenin/actin complex promotes microvessel normalization and suppresses tumor progression. J Exp Clin Cancer Res. 2019;38(1):285. doi:10.1186/s13046-019-1291-z

15. Xi X, Liu N, Wang Q, et al. ACT001, a novel PAI-1 inhibitor, exerts synergistic effects in combination with cisplatin by inhibiting PI3K/ AKT pathway in glioma. Cell Death Dis. 2019;10(10):757. doi:10.1038/s41419-019-1986-2

16. Darvishi N, Rahimi K, Mansouri K, et al. MiR-646 prevents proliferation and progression of human breast cancer cell lines by suppressing HDAC2 expression. Mol Cell Probes. 2020;53:101649. doi:10.1016/j.mcp.2020.101649

17. Arnold J, Engelmann JC, Schneider N, Bosserhoff AK, Kuphal S. miR-488-5p and its role in melanoma. Exp Mol Pathol. 2020;112:104348. doi:10.1016/j.yexmp.2019.104348

18. Peng Y, Liu YM, Li LC, Wang LL, Wu XL. microRNA-503 inhibits gastric cancer cell growth and epithelial-to-mesenchymal transition. Oncol Lett. 2014;7(4):1233-1238. doi:10.3892/ol.2014.1868

19. Dai X, Guo X, Liu J, et al. Circular RNA circGRAMD1B inhibits gastric cancer progression by sponging miR-130a-3p and regulating PTEN and p21 expression. Aging (Albany NY). 2019;11 (21):9689-9708. doi:10.18632/aging.102414

20. Wang KW, Dong M. Role of circular RNAs in gastric cancer: recent advances and prospects. World J Gastrointest Oncol. 2019;11 (6):459-469. doi:10.4251/wjgo.v11.i6.459

21. Huang S, Zhang X, Guan B, et al. A novel circular RNA hsa_circ_0008035 contributes to gastric cancer tumorigenesis through targeting the miR-375/YBX1 axis. Am J Transl Res. 2019;11 (4):2455-2462.

22. Liu P, Cai S, Li N. Circular RNA-hsa-circ-0000670 promotes gastric cancer progression through the microRNA-384/SIX4 axis. Exp Cell Res. 2020;394(2):112141.

23. Wang J, Lv W, Lin Z, Wang X, Bu J, Su Y. Hsa_circ_0003159 inhibits gastric cancer progression by regulating $\mathrm{miR}^{-}-223-3 \mathrm{p} /$ NDRG1 axis. Cancer Cell Int. 2020;20:57. doi:10.1186/s12935020-1119-0

24. Meng Q, Wang X, Xue T, Zhao Q, Wang W, Zhao K. Long noncoding RNA MIR99AHG promotes gastric cancer progression by inducing EMT and inhibiting apoptosis via miR577/FOXP1 axis. Cancer Cell Int. 2020;20:414. doi:10.1186/s12935-020-01510-6

25. Guo B, Wang W, Zhao Z, et al. Rab14 act as oncogene and induce proliferation of gastric cancer cells via AKT signaling pathway. PLoS One. 2017;12(1):e0170620. doi:10.1371/journal.pone.0170620

\section{Publish your work in this journal}

Cancer Management and Research is an international, peer-reviewed open access journal focusing on cancer research and the optimal use of preventative and integrated treatment interventions to achieve improved outcomes, enhanced survival and quality of life for the cancer patient.
The manuscript management system is completely online and includes a very quick and fair peer-review system, which is all easy to use. Visit http://www.dovepress.com/testimonials.php to read real quotes from published authors. 